\title{
Unveiling the omicron B.1.1. 529: The variant of concern that is rattling the globe
}

\author{
Ruby Dhar', Joyeeta Talukdar², Arnab Nayek ${ }^{3}$, Swati Ajmeriya ${ }^{4}$, Arun Kumar ${ }^{5}$, \\ Subhradip Karmakar ${ }^{6}$
}

${ }^{1}$ Scientist, ${ }^{2,3}$ Research Associate-I, ${ }^{6}$ Additional Professor, Department of Biochemistry, All India Institute of Medical Sciences, ${ }^{4}$ Senior Research Fellow, Indian Council of Medical Research, New Delhi, ${ }^{5}$ Professor, Department of Biochemistry, Jagannath Gupta Institute of Medical Sciences and Hospital, Budge Budge, Kolkata, West Bengal, India

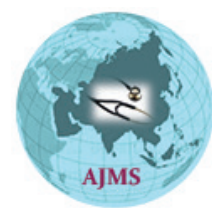

A B S T R A C T

Most viruses-including SARS-CoV-2, seem to have evolved over time. The lack of stringent proofreading mechanisms makes viral DNA/RNA replication error-prone. When a virus replicates, it sometimes changes a little bit, which is called mutations. Any virus with one or more new mutations can be referred to as a "variant" of the original virus. The last 2 years have witnessed the emergence of a large number of variants. Since the pandemic's beginning, the SARS-CoV-2 coronavirus has mutated extensively, resulting in the emergence of different variants of the virus. One of these is the delta variant (arising from Pango lineage B.1.617.2) that took the word in a storm this year (February-July). The current a variant of concern is the B.1.1.529 (Omicron) variant reported first from South Africa on November 24, 2021. In recent weeks, infections have been widely reported, along with the increased detection of the B.1.1.529 variant. We reviewed the emergence of the new variant (B1.1.529) and its possible outcomes.

Key words: COVID19; SARS-CoV2; Omicron; Pandemic; Variant of concern

Access this article online

Website:

http://nepjol.info/index.php/AJMS

DOI: 10.3126/ajms.v13i1.41117

E-ISSN: 2091-0576

P-ISSN: 2467-9100

Copyright (c) 2022 Asian Journal of Medical Sciences

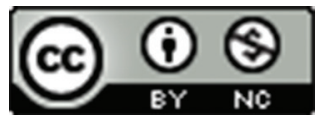

This work is licensed under a Creative Commons Attribution-NonCommercial 4.0 International License.

\section{INTRODUCTION}

With a population of 1.4 billion and a battling healthcare system after repeated COVID waves occurring within a year, India is waiting for a $3^{\text {rd }}$ imminent crisis looming right at its doorstep; ${ }^{1}$ the possible emergence of a new variant. A rapidly spreading variant of concern (VOC) is already dominant in S Africa and appears more transmissible than the most dominant delta variant. ${ }^{2}$

The nomenclature as recommended by the World Health Organization (WHO) Virus Evolution Working Group for naming SARS-CoV-2 genetic lineages by GISAID, Nextstrain, and Pango labeled this as the significant VOC. ${ }^{3}$

The alpha (Pango lineage B117), the beta (Pango lineage B1351), the gamma (Pango lineage P1), the epsilon
(Pango lineage 1.427-9), and the Delta (Pango lineage B1.617.2) were all categorized as VOC. VOC B.1.617.2, with an additional $\mathrm{K} 417 \mathrm{~K}$ mutation, the Delta variant Plus (B.1.617.2.1) was first identified in India in February 2021, was a variant of interest (VOI) in April 2021, quickly to become a VOC within a few weeks. Centers for Disease Control and Prevention has, however, already assigned the Delta variant as the VOC as it seems to have affected over $15 \%$ of its population. It is already the dominant variant in the UK. It is believed that Delta variant to be responsible for the horrific $2^{\text {nd }}$ COVID wave in the country, causing over $1 \mathrm{M}$ deaths. ${ }^{4}$

The US government interagency group developed a Variant Classification scheme that defines three classes of SARSCoV-2 variants: ${ }^{5}$ 
- The VOI

- The VOC

- The variant of high consequence.

VOI is a variant with specific genetic markers associated with receptor binding changes, reduced neutralization by antibodies generated against previous infection or vaccination, reduced efficacy of treatments, potential diagnostic impact, or predicted increase in transmissibility or disease severity. According to the WHO, a VOC are those variants for which there is evidence of an increase in transmissibility, more severe disease (e.g., increased hospitalizations or deaths), a significant reduction in neutralization by antibodies generated during previous infection or vaccination, reduced effectiveness of treatments or vaccines, or diagnostic detection failures. This delta variant seemed to be nearly upto $31 \%$ of India's COVID burden in its $2^{\text {nd }}$ wave.

\section{THE EMERGENCE OF OMICRON (B1.1.529)}

Excluding a few hotspots, the last couple of months witnessed a considerable decline in COVID cases worldwide. ${ }^{6}$ Things started to get normal with countries slowly reopening businesses and travel activities. The worldwide COVID-19 vaccination also set a new record with over 780 crores of doses administered and 350 crores ( $45 \%$ of the world population) fully vaccinated. India has set a new record, successfully vaccinating 120 crores of its population. ${ }^{7}$ While these developments were underway, and the virus seems to be under control, a new variant in South Africa reported just last week is changing the perception that the disease is far from over. South African health authorities seem to have identified a potentially more transmissible coronavirus variant. ${ }^{8}$ The WHO designated this newly identified coronavirus variant as B.1.1.529 and named Omicron. ' WHO's Technical Advisory Group on SARS-CoV-2 Virus Evolution (TAG-VE) labeled it a "VoC". In total, some 59 laboratory-confirmed cases of this new variant. Three were in Botswana, two were in Hong Kong, with the rest in South Africa, making Africa its site of origin. This variant seems to harbor a large number of spike protein mutations, surprisingly about 50 in total, with all clustered in one virus, making it one of the most heavily mutated versions of the virus seen to date. ${ }^{10}$ South African officials reported that more than 30 of the mutations were found in the spike protein, some of which were already present in the previous Delta variant (B 1617.2), with few additional new mutations specific to this. While the delta variant has mutations in the gene encoding the SARSCoV-2 spike protein causing the substitutions T478K, P681R, and L452R, Omicron seems to have additional changes making it even more transmissible. ${ }^{11}$ Given the large number of mutations it has accumulated just in a few generations, it likely evolved from immunocompromised persons, where the virus is not completely neutralized. This further necessity the importance of full vaccination for all. Given that Omicron has a large number of mutations, there are concerns that this virus might have enhanced transmissibility, contagiousness, immune evasion properties, and the ability to blunt the protective effect of vaccines. It is also reported that the $R$-value of this variant is $\sim$ two making it very transmissible.

It is just a matter of time that we will possibly know the full lethality of B 1.1.529. Until then, the best practice will be to adhere to risk mitigation protocols, strict genome surveillance, and vaccinate the unvaccinated populations.

\section{CONCLUDING REMARKS: EXCELLENT SCIENCE OR PUNISHMENT}

While South Africa is applauded for detecting the new variant, it is also at the receiving end from other nations worldwide, as seen by banning all movements from South Africa and quarantining the country. ${ }^{12}$ Nerveless Africa also has one of the world's least vaccinated, HIV-affected populations who are immune-compromised, making it a hotbed of the emergence of new variants. Omicron is assumed to carry an enormous mutation load making it immune evasive, more transmissible, and far more contagious than the delta variant. ${ }^{13,14}$ Until new sets of vaccines are steered to address these variants, the best bet we have is prevention and strict disease surveillance. An "Omni-vigilant" approach is needed to tackle the new "omicron" menace that is currently rattling the world.

\section{REFERENCES}

1. Second Wave of COVID-19 to Take a Toll on Indian Healthcare System: Fitch. Available from: https://www.business-standard. com/article/current-affairs/second-wave-of-covid-19-to-take-atoll-on-indian-healthcare-system-fitch-121041600297_1.html. [Last assessed on $2021 \mathrm{Apr} 16$ ].

2. The New Covid 'Omicron' Variant Has the Globe Freaking Out; 2021. Available from: https://www.rollingstone.com/politics/ politics-news/covid-omicron-variant-south-africa-fauci-1263440. [Last accessed on 2021 Nov 27].

3. WHO Announces Simple, Easy-to-Say Labels for SARS-CoV-2 Variants of Interest and Concern; 2021. Available from: https:// www.who.int/news/item/31-05-2021-who-announces-simpleeasy-to-say-labels-for-sars-cov-2-variants-of-interest-andconcern. [Last accessed on 2021 May 31].

4. Global COVID-19 Deaths Hit 5 Million as Delta Variant Sweeps the World; 2021. Available from: https://www.reuters.com/world/ global-covid-19-deaths-hit-5-million-delta-variant-sweepsworld-2021-10-02. [Last accessed on 2021 Oct 03].

5. SARS-CoV-2 Variant Classifications and Definitions; 2019. Available from: https://www.cdc.gov/coronavirus/2019-ncov/ 
variants/variant-info.html. [Last accessed on 2021 Dec 01].

6. COVID-19 Cases Showing a Declining Trend Across India, Says Government; 2021. Available from: https://www.thehindu. $\mathrm{com} /$ news/national/covid-19-cases-showing-a-declining-trendacross-india-says-government/article36503509.ece. [Last accessed on 2021 Sep 17].

7. India Crosses 120-Crore COVID-19 Vaccine Milestone; 2021. Available from: https://www.businesstoday.in/ coronavirus/story/india-crosses-120-crore-covid-19-vaccinemilestone-313443-2021-11-25. [Last accessed on 2021 Nov 25].

8. South African Scientists Brace for Wave Propelled by Omicron; 2021. Available from: https://www.apnews.com/article/coronaviruspandemic-joe-biden-science-health-lifestyle-83cad19ead1725137 bd7c3a371792ab7. [Last accessed on 2021 Nov 27].

9. WHO Labels New COVID Strain as Variant of Concern Amid Global Alarm; 2021. Available from: https://www.nbcnews.com/ news/world/who-meet-new-variant-covid-19-found-southernafrica-rcna6782. [Last accessed on 2021 Nov 26].

10. Heavily Mutated Omicron Variant Puts Scientists on Alert; 2021.
Available from: https://www.nature.com/articles/d41586-02103552-w. [Last accessed on 2021 Nov 25].

11. The Mutation that Helps Delta Spread Like Wildfire; 2021. Available from: https://www.nature.com/articles/d41586-02102275-2. [Last accessed on 2021 Aug 20].

12. South Africa Punished for Detecting Omicron COVID Variant: Government; 2021. Available from: https://www.economictimes. indiatimes.com/news/international/world-news/southafrica-punished-for-detecting-omicron-covid-variant-govt/ articleshow/87950027.cms. [Last accessed on 2021 Nov 27].

13. Is Omicron COVID Variant More Contagious than Delta Strain? Know the Symptoms, Risks Involved; 2021. Available from: https://www.thehealthsite.com/news/is-omicron-covid-variantmore-contagious-than-delta-strain-know-the-symptoms-risksinvolved-849156. [Last accessed on 2021 Nov 27].

14. New Omicron Variant Could be $500 \%$ More Infectious than Delta-Impossible to Contain; 2021. Available from: https://www. express.co.uk/news/world/1527814/omicron-variant-deltacoronavirus-who-covid-strain-mutant-uk-south-africa-latest-vn. [Last accessed on 2021 Nov 27].

\section{Authors' Contributions:}

RD- Drafted the manuscript with assistance from JT, AN and SA; AK- Provided critical comments and suggestions; AN- Assisted in statistical and data curation;

SK- Conceptualized and overseen the entire study.

Work attributed to:

Department of Biochemistry, All India Institute of Medical Sciences, New Delhi 110029, India

Orcid ID:

Dr. Ruby Dhar - (D) https://orcid.org/0000-0003-3600-6554

Dr. Joyeeta Talukdar - (D) https://orcid.org/0000-0002-4922-2283

Dr. Arun Kumar - (1) https://orcid.org/0000-0002-8800-0296

Dr. Subhradip Karmakar - (1) https://orcid.org/0000-0002-4757-8729

Arnab Nayek - (1) https://orcid.org/0000-0001-6767-8383

Swati Ajmeriya - (i) https://orcid.org/0000-0002-2015-0335

Source of Funding: We thank ICMR for providing fellowship to JT, AR and SA, Conflicts of Interest: None. 